\title{
Particle creation in the context of the emergent universe
}

\author{
B. Hamil \\ Département de TC de SNV, Université Hassiba Benbouali, Chlef, Algeria \\ M. Merad \\ Faculté des Sciences Exactes, Département des sciences de la matière, \\ Université de Oum El Bouaghi, 04000 Oum El Bouaghi, Algeria. \\ T. Birkandan \\ Department of Physics, Istanbul Technical University, 34469 Istanbul, Turkey.
}

Received 30 June 2020; accepted 11 November 2020

\begin{abstract}
We study the mechanism of particle creation in the context of the emergent universe (EU) scenario which is privileged by certain important characteristics such as the absence of time-like singularity. EU asymptotically coincides with an Einstein static model in the infinite past and it approaches to a de Sitter expansion phase at late times. By introducing the conformal time, we obtain the solution of the Klein-Gordon equation and by applying the "in" and "out" states method, the total number of produced particles and the total energy associated with them are determined.
\end{abstract}

Keywords: Schwinger effect; emergent universe scenarios; Klein-Gordon equation; bogoliubov transformation.

DOI: https://doi.org/10.31349/RevMexFis.67.219

\section{Introduction}

In recent years, there has been a great interest in the process of pair creation by strong electric fields after the pioneering works of Sauter [1], Heisenberg and Euler [2] and Schwinger [3]. Since then, the pair creation of particles and antiparticles from the unstable vacuum by external electromagnetic fields became a curious process in quantum electrodynamics (QED).

In a seminal study [3], Schwinger exactly calculated the pair creation probability from the vacuum due to an external field by applying the proper-time formulation. He showed that the pure magnetic fields do not produce any pairs of charged particles, but the presence of a magnetic field modifies the probability of pair creation when the vacuum is perturbed by an electric field. The probability of pair creation in a constant homogeneous electric field is given by the Schwinger rate $[4,5]$, namely,

$$
\mathcal{P} \propto e^{-\pi \frac{E_{c}}{E}}
$$

where $E_{c}=\left(m^{2} c^{3} / e \hbar\right) \simeq 10^{18} \mathrm{~V} / \mathrm{m}$ is Schwinger's critical field. This exponential is independent of the spacetime variables and does not admit a Taylor expansion in $e$ or $E$, which indicates that this is a nonperturbative effect.

In addition, the theory of quantized fields in curved spacetime is one way of unifying Einstein's general relativity and quantum field theory in Minkowski background. The mechanism of particle creation by the external electric field is analogous to the particle production by a time-dependent metric of a curved spacetime [6-9]. The cosmological particle creation mechanism in a time-dependent gravitational field is one of the most remarkable known results. This mechanism has an important role in explaining the origin of the structures in our Universe and opened a new field in physics.
In order to study the process of particle creation in a timedependent gravitational field, many different approaches have been developed such as the Hamiltonian diagonalization method $[10,11]$, path integral technique $[12,13]$, the Green function approach [14], the semiclassical WKB approximation $[15,16]$, the method based on vacuum-vacuum transition amplitude [3], and the "in" and "out" states formalism $[17,18]$.

Recently, the particle creation problem under the influence of electromagnetic and/or gravitational fields was studied in [19-27]. Also, more recently, the Schwinger effect by an SU(2) gauge field during inflation for scalar particles and fermions was calculated in [28] and [29,30].

On the other hand, one of the most serious questions in the standard cosmology is whether the universe has a beginning or has existed eternally, and this question has been leading us into a profound discussion by using general relativity and modern cosmology. The standard cosmological model implies that the universe stems from a Big Bang singularity. To elude this singularity, Ellis et al. [31,32] proposed an interesting model, called Emergent Universe (EU), in which the universe originates from an Einstein static state rather than a Big Bang singularity, and therefore, there is no time-like singularity.

In this paper, we study the mechanism of particle creation in a spatially closed Robertson-Walker model with the scale factor $a(t)=a_{0}+A e^{H_{0} t}$, where $a_{0}, A$ and $H_{0}$ are positive constants [33-35]. In this universe, there is no time-like singularity. It asymptotically coincides with the Einstein static model in the infinite past $a(t) \rightarrow a_{0}$ and, it approaches to a de Sitter expansion phase at late times $a(t) \rightarrow A e^{H_{0} t}$. In the beginning, we consider a spin- 0 scalar in spatially closed Robertson-Walker spacetime in the context of the emergent universe scenario. Then we solve the Klein-Gordon equation 
by introducing two successive changes of variables. In order to study the mechanism of particle creation, we use the canonical method based on Bogoliubov transformation connecting the "in" and "out" coefficients. This method allows us to find the number density of the created particles and the total energy.

\section{The Klein-Gordon equation}

To study the process of pair creation, we consider the case of spatially closed Robertson-Walker universe with the metric,

$$
d s^{2}=d t^{2}-a^{2}(t)\left[d \chi^{2}+\sin ^{2} \chi\left(d \theta^{2}+\sin ^{2} \theta d \varphi^{2}\right)\right],
$$

where $0 \leqslant \chi \leqslant \pi,, 0 \leq \theta<\pi, 0<\varphi<2 \pi$. The wave equation for a real massive scalar field which is coupled to the closed Robertson-Walker background, takes the form

$$
\frac{1}{\sqrt{-g}} \partial_{\mu}\left(g^{\mu \nu} \sqrt{-g} \partial_{\nu} \psi\right)+\left(m^{2}+\zeta R\right) \psi=0
$$

where $R=g^{\mu \nu} R_{\mu \nu}$ is the Ricci scalar and $\zeta$ is a numerical factor which takes the value $\zeta=0$ in the minimal coupling case and, $\zeta=1 / 6$ when a conformal coupling is considered. In this case, the equation of a massive scalar field is written as

$$
\begin{aligned}
& \left(\frac{\partial^{2}}{\partial t^{2}}+\frac{3 \dot{a}}{a} \frac{\partial}{\partial t}-\frac{1}{a^{2}(t)}\left[\frac{\partial^{2}}{\partial \chi^{2}}+2 \cot \chi \frac{\partial}{\partial \chi}-\frac{1}{\sin ^{2} \chi}\right.\right. \\
& \left.\left.\times\left\{\frac{\partial^{2}}{\partial \theta^{2}}+\cot \theta \frac{\partial}{\partial \theta}+\frac{1}{\sin ^{2} \theta} \frac{\partial^{2}}{\partial \varphi^{2}}\right\}\right]+m^{2}\right) \psi=0,
\end{aligned}
$$

where we have neglected the numerical factor $\zeta$ to yield minimal coupling (i.e. $\zeta=0$ ). To solve the differential equation (4), we write the solution $\psi(t, \chi, \theta, \varphi)$ in the form

$$
\psi(t, \chi, \theta, \varphi)=a^{-\frac{3}{2}}(t) Y^{n, \ell, \mu}(\chi, \theta, \varphi) \Psi(t),
$$

where $Y^{n, \ell, \mu}$ are the scalar harmonics on three-sphere $S^{3}$ and, $n \geqslant \ell \geqslant 0$ and $\ell \geqslant \mu \geqslant-\ell$. These harmonics can be expressed in terms of the standard $S^{2}$ scalar harmonics $\mathcal{Y}^{\ell, \mu}(\theta, \varphi)$ as

$$
Y^{n, \ell, \mu}(\chi, \theta, \varphi)=H^{n, \ell}(\chi) \mathcal{Y}^{\ell, \mu}(\theta, \varphi) .
$$

The eigenfunctions $H^{n, \ell}(\chi)$ satisfy the following differential equation $[36,37]$

$$
\begin{aligned}
& {\left[\frac{\partial^{2}}{\partial \chi^{2}}+2 \cot \chi \frac{\partial}{\partial \chi}-\frac{\ell(\ell+1)}{\sin ^{2} \chi}\right]} \\
& \quad \times H^{n, \ell}(\chi)=-n(n+2) H^{n, \ell}(\chi) .
\end{aligned}
$$

with

$$
H^{n, \ell}(\chi)=\sin ^{\ell} \chi C_{n-\ell}^{\ell+1}(\cos (\chi)),
$$

and $C_{n-\ell}^{\ell+1}(\cos (\chi))$ are Gegenbauer polynomials [36, 37]. The $S^{3}$ scalar harmonics $Y^{n, \ell, \mu}$ satisfy the following orthonormality conditions:

$$
\begin{aligned}
& \int Y^{n, \ell, \mu}(\chi, \theta, \varphi) Y^{* n^{\prime}, \ell^{\prime}, \mu^{\prime}}(\chi, \theta, \varphi) \sin ^{2} \chi d \chi \\
& \times \sin \theta d \theta d \varphi=\delta_{n n^{\prime}} \delta_{\ell \ell^{\prime}} \delta_{\mu \mu^{\prime}} .
\end{aligned}
$$

Substituting Eq. (5), (6) and (7) in Eq. (4), we obtain the differential equation for $\Psi(t)$,

$$
\left[\frac{\partial^{2}}{\partial t^{2}}+\omega_{n}^{2}(t)\right] \Psi(t)=0
$$

where

$$
\begin{aligned}
\omega_{n}^{2}(t) & =m^{2}-\frac{3}{2} \frac{A H_{0}^{2} e^{t H_{0}}}{a_{0}+A e^{H_{0} t}}-\frac{3}{4}\left(\frac{A H_{0} e^{t H_{0}}}{a_{0}+A e^{H_{0} t}}\right)^{2} \\
& +\frac{n(n+2)}{\left(a_{0}+A e^{H_{0} t}\right)^{2}} .
\end{aligned}
$$

The Eq. (10) involves $n$ and so, in general, the number of produced particles will depend on $n$. Equation (10), on the other hand, does not depend on the angular eingenvalues $\ell$, and so the number of produced particles are always independent of $\ell$ in closed Robertson-Walker spacetime. Note that Eq. (10) is similar to that of the harmonic oscillator with a time-dependent frequency.

Let us analyze the behavior of the time equation when $t \rightarrow \pm \infty$. In the asymptotic past $t \rightarrow-\infty$, Eq. (10) reduces to

$$
\begin{aligned}
{\left[\frac{\partial^{2}}{\partial t^{2}}+\omega_{n, i n}\right] \Psi(t) } & =0 \\
\sqrt{\frac{n(n+2)}{a_{0}^{2}}+m^{2}} & =\omega_{n, i n},
\end{aligned}
$$

with the solutions

$$
\Psi_{n, i n} \simeq \frac{e^{-i \omega_{n, i n} t}}{\sqrt{2 \omega_{n, i n}}} ; \quad \Psi_{n, i n}^{*} \simeq \frac{e^{i \omega_{n, i n} t}}{\sqrt{2 \omega_{n, i n}}} .
$$

On the other hand, in the asymptotic future $t \rightarrow+\infty$, Eq. (10) yields

$$
\left[\frac{\partial^{2}}{\partial t^{2}}+\omega_{\text {out }}\right] \Psi(t)=0 ; \quad \omega_{\text {out }}=\sqrt{m^{2}-\frac{9 H_{0}^{2}}{4}},
$$

with the solutions

$$
\Psi_{\text {out }}(t) \simeq \frac{e^{-i \omega_{\text {out }} t}}{\sqrt{2 \omega_{\text {out }}}} ; \quad \Psi_{\text {out }}^{*}(t) \simeq \frac{e^{i \omega_{\text {out }} t}}{\sqrt{2 \omega_{\text {out }}}} .
$$

We see that $\omega_{\text {out }}$ does not depend on the label $n$. This result is not surprising because in the future infinity we coincide with a de Sitter spacetime [38]. By making two successive changes of variables as

$$
\eta=\int \frac{d t}{a(t)}
$$


and $\xi=e^{H_{0} a_{0} \eta}$, the Eq. (10) takes the form

$$
\begin{aligned}
& \left((1-\xi) \xi \frac{d^{2}}{d \xi^{2}}+(1-2 \xi) \frac{d}{d \xi}+\frac{\frac{m^{2}}{H_{0}^{2}}-\frac{9}{4}}{(1-\xi)}\right. \\
& \left.+\frac{\frac{n(n+2)}{H_{0}^{2} a_{0}^{2}}+\frac{m^{2}}{H_{0}^{2}}}{\xi}-\left[\frac{n(n+2)}{a_{0}^{2} H_{0}^{2}}-\frac{3}{4}\right]\right) \Psi(\xi)=0 .
\end{aligned}
$$

The singularities of this differential equation are $\xi=0,1$ and $\infty$, and all are regular singularities. We can recognize this differential equation as a hypergeometric one by imposing the substitution,

$$
\Psi(\xi)=(1-\xi)^{\kappa} \xi^{v} \Xi(\xi)
$$

This, substituted into the differential equation, gives,

$$
\begin{aligned}
& \left(\frac{d^{2}}{d \xi^{2}}+(1+2 v-2(v+\kappa+1) \xi) \frac{d}{d \xi}\right. \\
& \left.-\left[\left(v+\kappa+\frac{1}{2}\right)^{2}+\frac{n(n+2)}{a_{0}^{2} H_{0}^{2}}-1\right]\right) \Xi(\xi)=0,
\end{aligned}
$$

where $\kappa=\left(i \omega_{\text {out }} / H_{0}\right)$ and $v=\left(i \omega_{n, i n} / H_{0}\right)$. The equation above is a hypergeometric equation whose solution around the origin $\xi=0$ is given in terms of the hypergeometric functions as [39]

$$
\begin{gathered}
\Xi_{1}=\mathbf{F}\left(\frac{1}{2}+v+\kappa+i \vartheta_{n},\right. \\
\left.\frac{1}{2}+v+\kappa-i \vartheta_{n}, 1+2 v ; \xi\right), \\
\Xi_{2}=\xi^{-2 v} \mathbf{F}\left(\frac{1}{2}-v+\kappa+i \vartheta_{n},\right. \\
\left.\frac{1}{2}-v+\kappa-i \vartheta_{n}, 1-2 v ; \xi\right),
\end{gathered}
$$

and the solution $\Psi(\xi)$ of Eq. (16) finally reads,

$$
\begin{aligned}
& \Psi_{1}=\xi^{v}(1-\xi)^{\kappa} \mathbf{F}( \frac{1}{2}+v+\kappa+i \vartheta_{n}, \\
&\left.\frac{1}{2}+v+\kappa-i \vartheta_{n}, 1+2 v ; \xi\right), \\
& \Psi_{2}=(1-\xi)^{\kappa} \xi^{-v} \mathbf{F}( \frac{1}{2}-v+\kappa+i \vartheta_{n}, \\
&\left.\frac{1}{2}-v+\kappa-i \vartheta_{n}, 1-2 v ; \xi\right),
\end{aligned}
$$

where $\vartheta_{n}=\sqrt{\left(n(n+2) / a_{0}^{2} H_{0}^{2}\right)-1}$. Using the invariance of Eq. (16) under the transformation $\xi \rightarrow 1-\xi$ and $\omega_{n, \text { in }} \rightarrow \omega_{\text {out }}$, we find another set of solutions

$$
\begin{aligned}
\Psi_{3}=(1-\xi)^{\kappa} \xi^{v} \mathbf{F}\left(\frac{1}{2}+v+\kappa+i \vartheta_{n},\right. \\
\left.\frac{1}{2}+v+\kappa-i \vartheta_{n}, 1+2 \kappa ; 1-\xi\right), \\
\Psi_{4}=\xi^{v}(1-\xi)^{-\kappa} \mathbf{F}\left(\frac{1}{2}+v-\kappa+i \vartheta_{n},\right. \\
\left.\frac{1}{2}-\kappa+v-i \vartheta_{n}, 1-2 \kappa, 1-\xi\right) .
\end{aligned}
$$

In the next section we will use the solutions of the field equation to analyze the mechanism of particle creation.

\section{Pair creation}

In order to study the process of particle creation, we will discuss the asymptotic behavior of the solutions of the KleinGordon equation when $t \rightarrow \pm \infty$, or equivalently when $\xi \rightarrow 0,1$. Firstly, when $t \rightarrow-\infty$ or $\xi \rightarrow 0$, we have

$$
\begin{aligned}
\lim _{\xi \rightarrow 0} \Psi_{1} & =\lim _{\xi \rightarrow 0} \frac{(1-\xi)^{\kappa} \xi^{v}}{\sqrt{2 \omega_{n}^{i n}}} \\
& \times \mathbf{F}(a, b, c ; \xi) \simeq \frac{e^{i \omega_{n, i n} t}}{\sqrt{2 \omega_{n, i n}}}, \\
\lim _{\xi \rightarrow 0} \Psi_{2} & =\lim _{\xi \rightarrow 0} \frac{(1-\xi)^{\kappa} \xi^{-v}}{\sqrt{2 \omega_{n, i n}}} \\
& \times \mathbf{F}\left(a^{\prime}, b^{\prime}, c^{\prime} ; \xi\right) \simeq \frac{e^{-i \omega_{n, i n} t}}{\sqrt{2 \omega_{n, i n}}},
\end{aligned}
$$

where $F(a, b, c ; 0)=1$. Then, as $t \rightarrow-\infty$, the positive and negative frequency modes are

$$
\Psi_{n, i n}^{*}=\Psi_{1} ; \quad \Psi_{n, i n}=\Psi_{2} .
$$

The modes (25), (26) may be used to define particle states and a Fock space in the Heisenberg picture in the standard way. In particular, the field modes $\Psi_{n}$ may be expanded

$$
\Psi_{n}=a_{n} \Psi_{n, i n}+a_{n}^{+} \Psi_{n, i n}^{*},
$$

where the operators $a_{n}, a_{n}^{+}$obey the commutation relations

$$
\left[a_{n}, a_{n^{\prime}}^{+}\right]=\delta_{n n^{\prime}},\left[a_{n}, a_{n^{\prime}}\right]=\left[a_{n}^{+}, a_{n^{\prime}}^{+}\right]=0 .
$$

Similarly, in the "out" region, when $t \rightarrow+\infty$ or $\xi \rightarrow 1$, we have

$$
\begin{aligned}
\lim _{\xi \rightarrow 0} \Psi_{n 3} & =\lim _{\xi \rightarrow 0} \frac{(1-\xi)^{\kappa} \xi^{v}}{\sqrt{2 \omega_{\text {out }}}} \\
& \times \mathbf{F}(a, b, c ; 1-\xi) \simeq \frac{e^{-i \omega_{\text {out }} t}}{\sqrt{2 \omega_{\text {out }}}}, \\
\lim _{\xi \rightarrow 0} \Psi_{n 4} & =\lim _{\xi \rightarrow 0} \frac{\xi^{v}(1-\xi)^{-\kappa}}{\sqrt{2 \omega_{\text {out }}}} \\
& \times \mathbf{F}\left(a^{\prime}, b^{\prime}, c^{\prime} ; 1-\xi\right) \simeq \frac{e^{i \omega_{\text {out }} t}}{\sqrt{2 \omega_{\text {out }}}},
\end{aligned}
$$


and the positive and negative frequency modes as $t \rightarrow+\infty$ are

$$
\Psi_{n 4}=\Psi_{n, \text { out }}^{*} ; \quad \Psi_{n 3}=\Psi_{n, \text { out }}
$$

The field modes $\Psi_{n}$ can be expanded in terms of these "out" modes as,

$$
\Psi_{n}=b_{n} \Psi_{n}^{\text {out }}+b_{n}^{+} \Psi_{n}^{* o u t}
$$

where

$$
\left[b_{n}, b_{n^{\prime}}^{+}\right]=\delta_{n n^{\prime}},\left[b_{n}, b_{n^{\prime}}\right]=\left[b_{n}^{+}, b_{n^{\prime}}^{+}\right]=0 .
$$

There are two different vacua $\left|0_{\text {in }}\right\rangle$ and $\left|0_{\text {out }}\right\rangle$ associated with two Fock spaces $\mathcal{F}^{\text {in }}$ and $\mathcal{F}^{\text {out }}$

$$
\left\{\begin{array}{rlrl}
a_{n}\left|0_{\text {in }}\right\rangle & =0, & & \forall n \\
b_{n}\left|0_{\text {out }}\right\rangle & =0, & & \forall n
\end{array} .\right.
$$

The creation and annhilation operators are related by the relations

$$
\left\{\begin{array}{l}
a_{n}=\alpha_{n} b_{n}+\beta_{n} b_{n}^{+} \\
b_{n}=\alpha_{n}^{*} a_{n}-\beta_{n}^{*} a_{n}^{+}
\end{array}\right.
$$

where $\alpha_{n}$ and $\beta_{n}$ are the Bogolioubov's coefficients. In order to obtain the density of created particles, we use the so called Bogoliubov transformation, connecting the "in" (at $t \rightarrow-\infty$ ) with the "out" (at $t \rightarrow+\infty$ ) states,

$$
\Psi_{n, \text { in }}=\alpha_{n} \Psi_{n, \text { out }}+\beta_{n} \Psi_{n, \text { out }}^{*} .
$$

The "in" state is written in terms of the outgoing positive and negative solutions and this is the origin of particle creation by a gravitational field. The coefficients $\alpha_{n}$ and $\beta_{n}$ are the Bogoliubov coefficients satisfying the bosonic relation given by

$$
\left|\alpha_{n}\right|^{2}-\left|\beta_{n}\right|^{2}=1 \text {. }
$$

By using the relation between the hypergeometric functions [39], namely,

$$
\begin{aligned}
\mathbf{F}(a, b, c, \varkappa) & =\frac{\Gamma(c) \Gamma(c-a-b)}{\Gamma(c-b) \Gamma(c-a)} \\
& \times \mathbf{F}(a, b, a+b-c+1,1-\varkappa) \\
& +\frac{\Gamma(c) \Gamma(a+b-c)}{\Gamma(b) \Gamma(a)}(1-\varkappa)^{c-a-b} \\
& \times \mathbf{F}(c-a, c-b, c-a-b+1,1-\varkappa),
\end{aligned}
$$

and

$$
\mathbf{F}(a, b, c, y)=(1-y)^{c-a-b} \mathbf{F}(c-a, c-b, c, y),
$$

we find that the Bogoliubov coefficients are

$$
\begin{gathered}
\alpha_{n}=\frac{\sqrt{\frac{\omega_{\text {out }}}{\omega_{n, \text { in }}}} \Gamma\left(1-2 i \frac{\omega_{n, \text { in }}}{H_{0}}\right) \Gamma\left(-2 i \frac{\omega_{\text {out }}}{H_{0}}\right)}{\Gamma\left(\frac{1}{2}-i \frac{\omega_{n, \text { in }}+i \omega_{\text {out }}}{H_{0}}+i \vartheta_{n}\right) \Gamma\left(\frac{1}{2}-i \frac{\omega_{n, \text { in }}+\omega_{\text {out }}}{H_{0}}-i \vartheta_{n}\right)}, \\
\beta_{n}=\frac{\sqrt{\frac{\omega_{\text {out }}}{\omega_{n, \text { in }}}} \Gamma\left(1-2 i \frac{\omega_{n, \text { in }}}{H_{0}}\right) \Gamma\left(2 i \frac{\omega_{\text {out }}}{H_{0}}\right)}{\Gamma\left(\frac{1}{2}-i \frac{\omega_{n, \text { in }}-\omega_{\text {out }}}{H_{0}}-i \vartheta_{n}\right) \Gamma\left(\frac{1}{2}-i \frac{\omega_{n, \text { in }}-\omega_{\text {out }}}{H_{0}}+i \vartheta_{n}\right)} .
\end{gathered}
$$

So, the number of particles is given by:

$$
\mathfrak{N}_{n}=\left\langle 0_{i n}\left|b_{n}^{+} b_{n}\right| 0_{i n}\right\rangle=\left|\beta_{n}\right|^{2}
$$

A direct calculation gives

$$
\mathfrak{N}_{n}=\frac{\cosh \pi\left(\frac{\omega_{n, \text { in }}-\omega_{\text {out }}}{H_{0}}-\vartheta_{n}\right) \cosh \pi\left(\frac{\omega_{n, \text { in }}-\omega_{\text {out }}}{H_{0}}+\vartheta_{n}\right)}{\sinh \frac{2 \pi \omega_{n, \text { in }}}{H_{0}} \sinh \frac{2 \pi \omega_{\text {out }}}{H_{0}}},
$$

where we used the relations,

$$
|\Gamma(i x)|^{2}=\frac{\pi}{x \sinh \pi x} ; \quad|\Gamma(1+i x)|^{2}=\frac{\pi x}{\sinh \pi x} ; \quad\left|\Gamma\left(\frac{1}{2}+i x\right)\right|^{2}=\frac{\pi}{\cosh \pi x} .
$$

The total number of the produced particles is obtained by taking the sum over all the oscillation modes as

$$
\mathfrak{N}=\sum_{n=0}^{\infty}(n+1)^{2} \frac{\cosh \pi\left(\frac{\omega_{n, \text { in }}-\omega_{\text {out }}}{H_{0}}-\vartheta_{n}\right) \cosh \pi\left(\frac{\omega_{n, \text { in }}-\omega_{\text {out }}}{H_{0}}+\vartheta_{n}\right)}{\sinh \frac{2 \pi \omega_{n, \text { in }}}{H_{0}} \sinh \frac{2 \pi \omega_{\text {out }}}{H_{0}}},
$$


and the total energy is given by

$$
E=\sum_{n=0}^{\infty} \omega_{\text {out }}(n+1)^{2} \frac{\cosh \pi\left(\frac{\omega_{n, \text { in }}-\omega_{\text {out }}}{H_{0}}-\vartheta_{n}\right) \cosh \pi\left(\frac{\omega_{n, \text { in }}-\omega_{\text {out }}}{H_{0}}+\vartheta_{n}\right)}{\sinh \frac{2 \pi \omega_{n, \text { in }}}{H_{0}} \sinh \frac{2 \pi \omega_{\text {out }}}{H_{0}}},
$$

where $(n+1)^{2}$ is the degree of degeneracy.

In Fig. 1, we plot the total number of produced particles $(\mathfrak{N})$ as a function of variable $M=m / H_{0}$ for several values of $h=a_{0} H_{0}$. The Eq. (46) has an infinite sum over all oscillation modes. Thus, we need to truncate the infinite series in order to perform a numerical study for the total number of particles. We calculated the sum for 100,1000 and 10000 terms and concluded that the number of particles increases with the number of terms taken into the summation indicating a divergence, while leaving the character of the curves unchanged. For 100, 1000 and 10000 terms, we have the number of particles in the numerical order $10^{4}, 10^{7 \sim 8}$ and $10^{10 \sim 11}$, respectively. In Fig. 1, we present the values obtained for the summation of 100 terms that shows the curves clearly. The number of produced particles decreases rapidly with $M$. Changes in the parameter $h$ change the behavior of the curves very slightly. While small values of $h$ produce individual curves, the curves start to coincide as $h$ increases. We did not include the curve for $h=10000$ for simplicity as it also coincides with the curves associated with $h=100$ and $h=1000$.

Indeed, the sums in Eqs. (46) and (47) are quite divergent, therefore the "in" and "out" representations are unitarily inequivalent, since Bogoliubov transformations need not to be unitary. Otherwise, it is remarkable that the system evolves slowly and continuously over time, which corresponds to the adiabatic regime. Consequently, the study of the frequency $\omega_{n}^{2}(t)$ in equations (10) and (11) implies that the asymptotic behavior of $\Psi(t)$ when $t \rightarrow \pm \infty$ is approximated by a WKB solution. Therefore, in order to regularize this divergence to find a sensible result, we consider that the process of pair production occurs at the time of maximum violation of the adiabatic condition, namely $(d / d t) \log \left|\left(\dot{\omega} / \omega^{2}\right)\right|=0$, around time $t_{c}$ and we can cut-off the sum at $n=N_{c}$, which is the highest value of $n$ [40-42]. Then the expressions of the total number $\mathfrak{N}$ of particles and the total energy are written according to $N_{c}$ as follows

$$
\begin{gathered}
\mathfrak{N}=\sum_{n=0}^{N_{c}}(n+1)^{2} \frac{\cosh \pi\left(\frac{\omega_{n, \text { in }}-\omega_{\text {out }}}{H_{0}}-\vartheta_{n}\right) \cosh \pi\left(\frac{\omega_{n, \text { in }}-\omega_{\text {out }}}{H_{0}}+\vartheta_{n}\right)}{\sinh \frac{2 \pi \omega_{n, \text { in }}}{H_{0}} \sinh \frac{2 \pi \omega_{\text {out }}}{H_{0}}}, \\
E=\sum_{n=0}^{N_{c}} \omega_{\text {out }}(n+1)^{2} \frac{\cosh \pi\left(\frac{\omega_{n, \text { in }}-\omega_{\text {out }}}{H_{0}}-\vartheta_{n}\right) \cosh \pi\left(\frac{\omega_{n, \text { in }}-\omega_{\text {out }}}{H_{0}}+\vartheta_{n}\right)}{\sinh \frac{2 \pi \omega_{n, \text { in }}}{H_{0}} \sinh \frac{2 \pi \omega_{\text {out }}}{H_{0}}},
\end{gathered}
$$

and their asymptotic behaviours are given respectively by

$$
\mathfrak{N} \simeq \frac{1}{3} \frac{N_{c}^{3}}{\sinh \left(\frac{2 \pi \omega_{\text {out }}}{H_{0}}\right)}
$$

and

$$
E \simeq \frac{1}{3} \frac{N_{c}^{3} \omega_{\text {out }}}{\sinh \left(\frac{2 \pi \omega_{\text {out }}}{H_{0}}\right)}
$$

Finally, let us study the limit $a_{0} \rightarrow 0$ that reproduces the de Sitter case. By taking into account that

$$
n(n+2) \rightarrow k^{2}, H_{0} \rightarrow H \text { and } \lim _{a_{0} \rightarrow 0} \omega_{n, i n}=\frac{|k|}{a_{0}}, \lim _{a_{0} \rightarrow 0} \vartheta_{n}=\frac{|k|}{H a_{0}},
$$

where $k^{2}=k_{x}^{2}+k_{y}^{2}+k_{n}^{2}$. For these conditions, Eq. (44) becomes

$$
\mathfrak{N}_{a_{0} \rightarrow 0}=\lim _{a_{0} \rightarrow 0} \frac{\cosh \pi\left(\sqrt{\frac{m^{2}}{H}-\frac{9}{4}}\right) \cosh \pi\left(\frac{2|k|}{H a_{0}}-\sqrt{\frac{m^{2}}{H}-\frac{9}{4}}\right)}{\sinh \left(\frac{2 \pi|k|}{H a_{0}}\right) \sinh \left(2 \pi \sqrt{\frac{m^{2}}{H}-\frac{9}{4}}\right)} .
$$

By using of the formula

we obtain

$$
\lim _{z \rightarrow+\infty} \sinh z \rightarrow e^{z} ; \lim _{z \rightarrow+\infty} \cosh z \rightarrow e^{z}
$$

$$
\mathfrak{N}_{a_{0} \rightarrow 0}=\left[e^{2 \pi \sqrt{\frac{m^{2}}{H}-\frac{9}{4}}}-1\right]^{-1}
$$




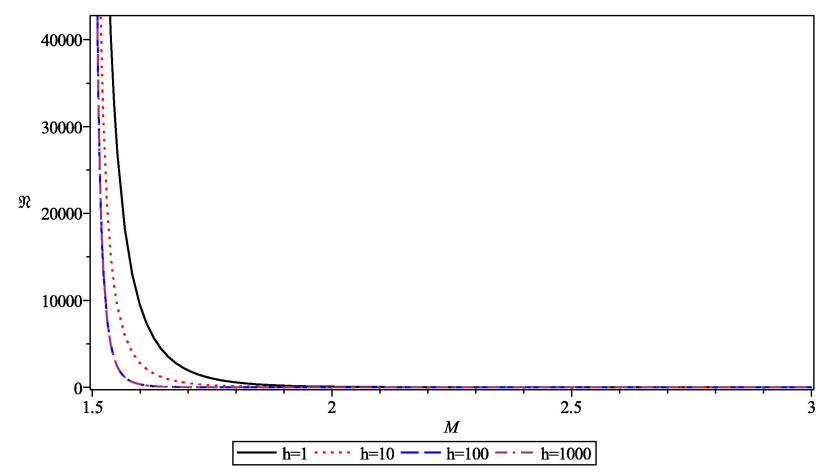

FigURE 1. ( $\mathfrak{N}$ vs. $\left.M=\frac{m}{H_{0}}\right)$ for some $\mathrm{h}=a_{0} H_{0}$ values).

and this result agrees exactly with that of [43]. Integrating this expression over all wave modes gives a divergent result,

$$
\frac{1}{(2 \pi)^{3}} \int d^{3} k \mathfrak{N}_{a_{0} \rightarrow 0}=\frac{\mathfrak{N}_{a_{0} \rightarrow 0}}{(2 \pi)^{2}} \int_{0}^{+\infty} k^{2} d k .
$$

We introduce $k_{c}$ as the highest value of the momentum for which the pairs have been created at a given time [40-42]. Consequently, the total number of created particles is given by

$$
\frac{1}{(2 \pi)^{3}} \int d^{3} k \mathfrak{N}_{a_{0} \rightarrow 0}=\frac{k_{c}^{3} \mathfrak{N}_{a_{0} \rightarrow 0}}{6 \pi^{2}}
$$

\section{Conclusion}

We studied an explicit calculation of the Klein-Gordon equation in spatially closed Robertson-Walker universe with the emergent universe (EU) scenarios whose scale factor evolution is modeled by $a(t)=a_{0}+A e^{H_{0} t}$. It is remarkable that this universe is characterized by the absence of the time-like singularity and, it asymptotically coincides with the Einstein static model in the infinite past and it approaches to a de Sitter expansion phase at late times.

The exact solution of the Klein-Gordon equation is obtained in terms of the hypergeometric functions for the temporal part, multiplied by the scalar harmonics on three-sphere $S^{3}$. To calculate the total number of produced particles and the total energy associated with them, we analyzed the asymptotic behavior of the solutions of the Klein-Gordon equation when $t \rightarrow \pm \infty$ in order to determine the suitable choice of the "in" and "out" states. Consequently, we applied the usual method of Bogoliubov transformations to find the coefficients connecting the "in" and "out" states that directly yield our results. We plotted the number of produced particles using a truncated series as a function of $m / H_{0}$ to see that the number of produced particles decreases rapidly with $m / H_{0}$. Finally, we studied the limit $a_{0} \rightarrow 0$ that reproduced the de Sitter case as expected.
1. F. Sauter, Über das Verhalten eines Elektrons im homogenen elektrischen Feld nach der relativistischen Theorie Diracs, Z. Phys. 69 (1931) 742, https://doi.org/10.1007/ $\mathrm{BF} 01339461$.

2. W. Heisenberg and H. Euler, arXiv:physics/0605038.

3. J. Schwinger, On Gauge Invariance and Vacuum Polarization, Phys. Rev. 82 (1951) 664, https://doi.org/10.1103/ PhysRev.82.664

4. S. P. Gavrilov and D. M. Gitman, Vacuum instability in external fields, Phys. Rev. D 53 (1996) 7162, https://doi. org/10.1103/PhysRevD.53.7162

5. A. I. Nikishov, Barrier scattering in field theory removal of Klein paradox, Nucl. Phys. B 21 (1970) 346, https : / / doi. org/10.1016/0550-3213(70) 90527-4

6. L. Parker, Particle Creation in Expanding Universes, Phys. Rev. Lett. 21 (1968) 562, https://doi.org/10.1103/ PhysRevLett.21.562

7. L. Parker, Quantized Fields and Particle Creation in Expanding Universes. I, Phys. Rev. 183 (1969) 1057, https : / / doi. org/10.1103/PhysRev.183.1057

8. L. Parker, Quantized Fields and Particle Creation in Expanding Universes. II, Phys. Rev. D 3 (1971) 346, https : / / doi . org/10.1103/PhysRevD.3.346

9. E. Mottola, Particle creation in de Sitter space, Phys. Rev. D 31 (1985) 754, https://doi.org/10.1103/PhysRevD. 31.754
10. A. A. Grib, S. G. Mamayev, and V. M. Mostepanenko, Particle creation from vacuum in homogeneous isotropic models of the Universe, Gen. Relat. Gravit. 7 (1976) 535, https: //doi.org/10.1007/BF00766413

11. A. A. Grib, S. G. Mamayev, and V. M. Mostepanenko, Vacuum quantum effects in strong fields (Friedmann Laboratory Pub., St. Petersburg, 1994).

12. D. M. Chitre and J. B. Hartle, Path-integral quantization and cosmological particle production: An example, Phys. Rev. D 16 (1977) 251, https://doi.org/10.1103/PhysRevD . 16.251

13. J. B. Hartle and S. W. Hawking, Path-integral derivation of black-hole radiance, Phys. Rev. D 13 (1976) 2188, https: //doi.org/10.1103/PhysRevD.13.2188.

14. S. P. Gavrilov, D. M. Gitman, and S. D. Odintsov, Quantum Scalar Field in the FRW Universe with a Constant Electromagnetic Background, Int. J. Mod. Phys. A 12 (1997) 4837, https://doi.org/10.1142/S0217751X97002589

15. S. Biswas, J. Guha, and N. G. Sarkar, Particle production in de Sitter space, Class. Quantum Grav. 12 (1995) 1591, https : //doi.org/10.1088/0264-9381/12/7/005.

16. S. Biswas, A. Shaw, and B. Modak, Schoringer result on particle production from complex paths WKB approximation, Int. J. Mod. Phys. A 15 (2000) 3717, https://doi.org/10. 1142/S0217751X00001221 
17. V. M. Villalba and W. Greiner, Creation of scalar and Dirac particles in the presence of a time varying electric field in an anisotropic Bianchi type I universe, Phys. Rev. D 65 (2001) 025007, https://doi.org/10.1103/PhysRevD.65. 025007 .

18. V. M. Villalba, Creation of scalar particles in the presence of a constant electric field in an anisotropic cosmological universe, Phys. Rev. D 60 (1999) 127501, https://doi.org/10. $1103 /$ PhysRevD.60.127501

19. E. Bavarsad, S. P. Kim, C. Stahl, and S.-S. Xue, Effect of a magnetic field on Schwinger mechanism in de Sitter spacetime, Phys. Rev. D 97 (2018) 025017, https://doi.org/10. 1103/PhysRevD.97.025017

20. T. Kobayashi and N. Afshordi, Schwinger effect in 4D de Sitter space and constraints on magnetogenesis in the early universe, J. High Energy Phys. 2014 (2014) 166, https : / / doi .org/ $10.1007 /$ JHEP10 (2014) 166

21. C. Stahl and S.-S. Xue, Schwinger effect and backreaction in de Sitter spacetime, Phys. Lett. B 760 (2016) 288, https: //doi.org/10.1016/j.physletb.2016.07.011.

22. M. B. Fröb et al., , Schwinger effect in de Sitter space, J. Cosmol. Astropart. Phys. 2014 (2014) 009, https: / / doi .org/ 10.1088/1475-7516/2014/04/009

23. T. Hayashinaka, T. Fujita, and J. Yokoyama, Fermionic Schwinger effect and induced current in de Sitter space, J. Cosmol. Astropart. Phys. 2016 (2016) 010, https: //doi .org/ 10.1088/1475-7516/2016/07/010

24. S. P. Gavrilov, D. M. Gitman, and A. A. Shishmarev, Pair production from the vacuum by a weakly inhomogeneous space-dependent electric potential, Phys. Rev. D 99 (2019) 116014, https://doi.org/10.1103/PhysRevD.99. 116014 .

25. S. P. Gavrilov, D. M. Gitman, and A. A. Shishmarev, Particle scattering and vacuum instability by exponential steps, Phys. Rev. D 96 (2017) 096020, https://doi.org/10.1103/ PhysRevD.96.096020

26. B. Hamil and M. Merad, Schwinger mechanism on de Sitter background, Int. J. Mod. Phys. A 33 (2018) 1850177, https : //doi.org/10.1142/S0217751X18501774.

27. B. Hamil, Pair creation of neutral Dirac particle in $(1+2) d$ noncommutative spacetime, Mod. Phys. Lett. A 33 (2018) 1850017, https://doi.org/10.1142/S0217732318500177.

28. K. D. Lozanov, A. Maleknejad, and E. Komatsu, Schwinger effect by an $S U(2)$ gauge field during inflation, J. High Energy Phys. 2019 (2019) 41, https://doi.org/10. $1007 /$ JHEP02 (2019) 041

29. L. Mirzagholi, A. Maleknejad, and K. D. Lozanov, Production and backreaction of fermions from axion- $S U(2)$ gauge fields during inflation, Phys. Rev. D 101 (2020) 083528, https: //doi.org/10.1103/PhysRevD.101.083528.

30. V. Domcke, Y. Ema, K. Mukaida, and R. Sato, Chiral anomaly and Schwinger effect in non-abelian gauge theories, J. High Energy Phys. 2019 (2019) 111, https://doi.org/10. 1007 / JHEP 03 (2019) 111

31. G. F. R. Ellis, J. Murugan, and C. G. Tsagas, The emergent universe: an explicit construction, Class. Quantum Gravit. 21 (2003) 233, https://doi.org/10.1088/ 0264-9381/21/1/016.

32. G. F. R. Ellis and R. Maartens, The emergent universe: inflationary cosmology with no singularity, Class. Quantum Gravit. 21 (2003) 223, https://doi.org/10.1088/ 0264-9381/21/1/015.

33. P. Labraña, Emergent universe scenario and the low CMB multipoles, Phys. Rev. D 91 (2015) 083534, https://doi. org/10.1103/PhysRevD.91.083534

34. C. Ríos, P. Labraña, and A. Cid, The Emergent Universe and the Anomalies in the Cosmic Microwave Background, J. Phys.: Conf. Ser. 720 (2016) 012008, https://doi.org/10. 1088/1742-6596/720/1/012008

35. P. Labraña, Emergent Universe Scenario and the Low CMB Multipoles, J. Phys.: Conf. Ser. 720 (2016) 012016, https : //doi.org/10.1088/1742-6596/720/1/012016

36. L. Lindblom, N. W. Taylor, and F. Zhang, Scalar, vector and tensor harmonics on the three-sphere, Gen. Relativ. Gravit. 49 (2017) 139, https://doi.org/10.1007/ s10714-017-2303-y

37. V. D. Sandberg, Tensor spherical harmonics on $S^{2}$ and $S^{3}$ as eigenvalue problems, J. Math. Phys. 19 (1978) 2441, https: //doi.org/10.1063/1.523649

38. J. Garriga, Pair production by an electric field in $(1+1)$ dimensional de Sitter space, Phys. Rev. D 49 (1994) 6343, https://doi.org/10.1103/PhysRevD.49.6343.

39. I. S. Gradshteyn and I. M. Ryzhik, Table of Integrals, Series, and Products, 4th ed. (Academic Press, New York, 1980).

40. T. Kobayashia and N. Afshordi, JHEP10, 166 (2014).

41. M. B. Fröb et al., , Schwinger effect in de Sitter space, J. Cosmol. Astropart. Phys. 2014 (2014) 009, https : / / doi . org/ $10.1088 / 1475-7516 / 2014 / 04 / 009$

42. M. Banyeres, G. Domènech, and J. Garriga, Vacuum birefringence and the Schwinger effect in $(3+1)$ de Sitter, J. Cosmol. Astropart. Phys. 2018 (2018) 023, https: / / doi.org/10. 1088/1475-7516/2018/10/023

43. T. Markkanen and A. Rajantie, Massive scalar field evolution in de Sitter, J. High Energy Phys. 2017 (2017) 133, https: //doi.org/10.1007/JHEP01 (2017)133 